\title{
Examples of buildings constructed via covering spaces
}

\author{
Michael W. Davis *
}

Abstract. Covering space theory is used to construct new examples of buildings.

Mathematics Subject Classification (2000). 20F65, 20F55, 20E42, 20J06, 51E24, 57M07.

Keywords. Building, Coxeter group.

\section{Introduction}

A building consists of a Coxeter system $(W, S)$, a set $\ell$ (of "chambers") and a "Weyl distance" $\delta: \varphi \times \ell \rightarrow W$, satisfying certain axioms. Given a building $\ell$, a space $X$, and a "mirror structure" on $X$, one can construct a "realization" $U(\mathcal{C}, X)$ of the

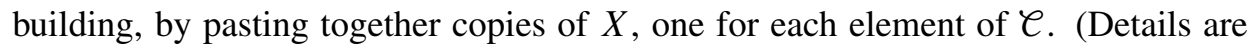
given in $§ 1.2$.) The classical choice for $X$ is a simplex.

The following types of buildings have been studied:

(1) spherical buildings, where $W$ is a finite Coxeter group;

(2) affine buildings, where $W$ is a Euclidean reflection group;

(3) hyperbolic buildings, where $W$ is a hyperbolic reflection group (or possibly a word hyperbolic Coxeter group), cf. [5], [6], [7], [17], [18], [19], [20], [21], [27], [30], [31];

(4) Kac-Moody buildings over finite fields, where $W$ is fairly arbitrary but where the "thickness" is restricted to be a constant prime power $q$, cf. [9], [24], [29];

(5) right-angled buildings, where $W$ is required to be a right-angled Coxeter group, cf. [4], [11], [12], [17], [26].

Classical buildings are either spherical or affine. There is some overlap between the three classes of "nonclassical" buildings; however, each class contains examples which are distinct from the other two.

The purpose of this note is to use covering space theory to construct new examples of buildings. On the level of Coxeter systems, this construction was described

\footnotetext{
*The author was partially supported by NSF grant DMS 0706259.
} 
previously in [10]. It goes as follows. Start with a Coxeter system $\left(W^{\prime}, S\right)$. Change some $m(s, t)$ 's in its Coxeter matrix from integers $\geq 2$ to the symbol $\infty$. This defines a new Coxeter system $(W, S)$. Suppose that $\ell^{\prime}$ is a building of type $\left(W^{\prime}, S\right)$. We want to use it construct a building $\mathcal{C}$ of type $(W, S)$. Let $K$ be the "Davis chamber" for $(W, S)$. Use it to get a realization $U\left(\ell^{\prime}, K\right)$ for $\ell^{\prime}$. Since we made changes in $W^{\prime}$, the space $U\left(\complement^{\prime}, K\right)$ will not be simply connected. However, as we show in Theorem 2.5, its universal cover is the realization of a building $\mathcal{C}$ of type $(W, S)$.

Although in these constructions we can start with any building $\mathcal{C}^{\prime}$, many interesting examples can be constructed by starting with the case where $W^{\prime}$ is a finite Coxeter group and $\ell^{\prime}$ is a spherical building. In particular, there are examples of buildings with the following features.

- There are buildings whose maximal spherical residues contain an arbitrary set of spherical buildings and whose standard realizations are highly connected at infinity and so, cannot be obtained by the procedure of simply taking the free product of various spherical buildings. (The concept of a "free product" of buildings is defined in Example 3.3 at the end of §3.1.)

- In contrast to the (irreducible) Kac-Moody case, there are examples of buildings with different thicknesses for different types of spherical residues. In other words, even when these spherical residues are associated to algebraic groups over finite fields, the fields may be different. (Previous examples of this in the case of 2-dimensional hyperbolic buildings had been noticed in [6].)

- When $\mathcal{C}^{\prime}$ is a finite spherical building, the automorphism group Aut $(\mathcal{C})$ always contains a torsion-free uniform lattice.

In $\S 3$ we specialize to the case where $\ell^{\prime}$ is a direct product, $\ell_{0} \times \cdots \times \ell_{p}$. By making appropriate choices of which $m(s, t)$ 's to change to $\infty$, we can define the notions of "free products" and "graph products" of buildings analogously to the corresponding notions for groups. When each of the buildings $\ell_{i}$ has the same rank, there also is the notion of a "square product," $\ell_{0} \square \cdots \square \ell_{p}$ (see Example 3.2). The square product is interesting when there are only two factors, $\ell_{0}, \ell_{1}$, and both are spherical buildings. In this case the fundamental chamber $K$ is combinatorially isomorphic to an $n$-cube. The link of one vertex of $K$ in the standard realization, $\mathcal{U}\left(\mathcal{C}_{0} \square \mathcal{C}_{1}, K\right)$ is the spherical realization of $\mathcal{C}_{0}$ while the link at an opposite vertex is the spherical realization of $\ell_{1}$ (see Example 3.9). This construction can be used to get new examples of 3-dimensional hyperbolic buildings (see Examples 3.10 and 3.11).

My thanks go to Tadeusz Januszkiewicz for several helpful conversations concerning these examples during the past twenty years. Thanks also to the referee for his careful proofreading and for several valuable suggestions. 


\section{Basic definitions}

1.1. Coxeter groups. A Coxeter matrix over a set $S$ is an $S \times S$ symmetric matrix $M=(m(s, t))$ with each diagonal entry equal to 1 and each off-diagonal entry either an integer $\geq 2$ or the symbol $\infty$. The matrix $M$ defines a presentation of a group $W$ as follows: the set of generators is $S$ and the relations have the form $(s t)^{m(s, t)}$ where $(s, t)$ ranges over all pairs in $S \times S$ such that $m(s, t) \neq \infty$. The pair $(W, S)$ is a Coxeter system (cf. [1], [8], [12]). Given $T \subset S, W_{T}$ denotes the subgroup generated by $T$; it is called a special subgroup. The pair $\left(W_{T}, T\right)$ is itself a Coxeter system (see [8], IV.8, Theorem 2 (i)). The subset $T$ is spherical if $W_{T}$ is finite.

Definition 1.1. The poset of spherical subsets of $S$ (partially ordered by inclusion) is denoted $S$ (or $S(W, S)$ ). The set of nonempty elements in $S$ is an abstract simplicial complex $L$ (or $L(W, S)$ ) called the nerve of $(W, S)$. (In other words, the vertex set of $L(W, S)$ is $S$ and a nonempty subset $T \subset S$ spans a simplex if and only if it is spherical.)

Given a word $s=\left(s_{1}, \ldots, s_{n}\right)$ in $S$, its value, $w(s)$, is the element of $W$ defined by $w(s):=s_{1} \cdots s_{n}$. The word $s$ is a reduced expression if it is a word of minimum length for $w(s)$, i.e., if $l(w(s))=n$.

Tits' solution to the word problem. Given elements $s, t \in S$ and an integer $m \geq 2$, let $\operatorname{prod}(s, t ; m)$ denote the alternating word $(s, t, \ldots)$ in $s$ and $t$ beginning with $s$ and having length $m$. Consider the following two operations on a word $s$ :

(I) delete a subword of the form $s s$;

(II) replace a subword $\operatorname{prod}(s, t ; m(s, t))$ by $\operatorname{prod}(t, s ; m(s, t))$.

An operation of type (I) is a cancellation; one of type (II) is a flip.

Tits proved that if a word $s=\left(s_{1}, \ldots, s_{n}\right)$ is not a reduced expression for an element $w$, then it can be changed to a reduced expression for the same element by a sequence of flips and cancellations. Moreover, if $\boldsymbol{s}$ and $\boldsymbol{s}^{\prime}$ are two reduced expressions for the same element, then one can be changed to the other by a sequence of flips (see $[1], \S 2.3 .3)$.

\subsection{Buildings}

Definition 1.2 (cf. [1], [25]). Suppose that $(W, S)$ is a Coxeter system. A building of type $(W, S)$ is a pair $(\mathcal{C}, \delta)$ consisting of a nonempty set $\mathcal{C}$ (the elements of which are called chambers), and a function $\delta: \varphi \times \varphi \rightarrow W$ (called the Weyl distance) so that the following conditions hold for all $C, D \in \mathcal{C}$ :

(WD1) $\delta(C, D)=1$ if and only if $C=D$. 
(WD2) If $\delta(C, D)=w$ and $C^{\prime} \in \mathcal{C}$ satisfies $\delta\left(C^{\prime}, C\right)=s \in S$, then $\delta\left(C^{\prime}, D\right)=$ $s w$ or $w$. If, in addition, $l(s w)=l(w)+1$, then $\delta\left(C^{\prime}, D\right)=s w$.

(WD3) If $\delta(C, D)=w$, then for any $s \in S$ there is a chamber $C^{\prime} \in \mathcal{C}$ such that $\delta\left(C^{\prime}, C\right)=s$ and $\delta\left(C^{\prime}, D\right)=s w$.

Example 1.3. The group $W$ itself has the structure of a building: $\delta: W \times W \rightarrow W$ is defined by $\delta(v, w)=v^{-1} w$. It is called the thin building of type $(W, S)$.

A chamber system is a set $\mathcal{C}$ together with a family of equivalence relations on $\mathcal{C}$ indexed by another set $S$. Chambers $C, D \in \mathcal{C}$ are $s$-adjacent if they are $s$-equivalent and not equal. A gallery in $C$ is a finite sequence of chambers $\left(C_{0}, \ldots, C_{k}\right)$ such that $C_{j-1}$ is adjacent to $C_{j}$, for $1 \leq j \leq k$. The type of this gallery is the word $\boldsymbol{s}=\left(s_{1}, \ldots, s_{k}\right)$ where $C_{j-1}$ is $s_{j}$-adjacent to $C_{j}$. If each $s_{j}$ belongs to a given subset $T$ of $S$, then the gallery is a $T$-gallery. Two chambers are in the same $T$-connected component if they can be connected by a $T$-gallery. The $T$-connected components of a chamber system $C$ are its residues of type $T$. An $s$-equivalence class is the same thing as a residue of type $\{s\}$. (An $s$-equivalence class is sometimes called a panel.)

Now suppose that $\ell$ is a building of type $(W, S)$. Chambers $C, D \in \bigodot$ are $s$ adjacent if $\delta(C, D)=s$; they are $s$-equivalent if they are either $s$-adjacent or equal. This gives $\ell$ the structure of a chamber system. When $\ell=W$, a $T$-residue is just a left coset of $W_{T}$.

A residue of type $T$ in a building is itself a building; its type is $\left(W_{T}, T\right)$. A building of type $(W, S)$ is spherical if $W$ is finite. A building has finite thickness if each $s$-equivalence class is finite, for each $s \in S$. (This implies all spherical residues are finite.) Henceforth, all buildings will be assumed to have finite thickness.

A gallery $\left(C_{0}, \ldots, C_{k}\right)$ of type $s$ is reduced if $s$ is a reduced expression for $w(s)$.

It is proved in [1], Proposition 5.23, that the conditions (WD1), (WD2), (WD3) in Definition 1.2 are equivalent to the following two conditions on a chamber system $\varphi$, equipped with a function $\delta: \varphi \times \ell \rightarrow W$.

- Each $s$-equivalence class has at least two elements.

- Given a reduced expression $s$ for an element $w \in W$, there is a gallery of type $s$ from $C$ to $D$ if and only if $\delta(C, D)=w$.

(This is the definition in [25].)

An apartment in $\mathcal{C}$ is a subset which is $W$-isometric to the thin building $W$. In other words, it is a subset $\mathcal{A} \subset \mathcal{C}$ such that for any $C \in \mathcal{A}$, the function $\rho_{C}: \mathcal{A} \rightarrow W$ defined by $D \mapsto \delta(C, D)$ is an isomorphism.

An automorphism of $C$ is a self-bijection which preserves $s$-equivalence classes for each $s \in S$. Equivalently, it is a self-bijection which preserves Weyl distance.

Given $C \in \mathcal{C}$, the combinatorial ball of radius $n$ about $C$ is the set $B_{C}(n):=$ $\{D \in \mathcal{C} \mid l(\delta(C, D)) \leq n\}$. There is a natural topology on the group Aut $(\mathcal{C})$ of automorphisms of $\mathcal{C}$ : an open neighborhood of $1 \in \operatorname{Aut}(\mathcal{C})$ is the set of automorphisms 
which fix each element of $B_{C}(n)$ for some $n \in \mathbb{N}$ and $C \in \mathcal{C}$. (The neighborhood is small if $n$ is large.) Since $\mathcal{C}$ is locally finite, $\operatorname{Aut}(\mathcal{C})$ is a locally compact, totally disconnected topological group. As such, it has a Haar measure. A closed subgroup $G \subset \operatorname{Aut}(\mathcal{C})$ inherits a topology and a Haar measure. A subgroup $\Gamma \subset G$ is a lattice if it is discrete and $G / \Gamma$ has finite volume. It is a uniform lattice if $G / \Gamma$ is compact. A discrete subgroup $\Gamma \subset \operatorname{Aut}(\mathcal{C})$ is a uniform lattice if and only if $\mathcal{C} \Gamma$ is finite. (See [26] for a discussion of lattices in $\operatorname{Aut}(\mathcal{C})$, when $\mathcal{C}$ is a right angled building.)

Definition 1.4. A subgroup $G \subset \operatorname{Aut}(\mathcal{C})$ is chamber-transitive if it is transitive on $\mathcal{C}$. It is strongly transitive if it is transitive on the set of pairs $(\mathcal{A}, C)$, where $\mathcal{A}$ is an apartment in $\mathcal{C}$ and $C \in \mathcal{A}$ (cf. [1], §6.1.1). (In fact, it is not necessary use all apartments in this definition, $\mathcal{A}$ need only belong to a certain "system of apartments" satisfying the classical axioms for a building, cf. [1], §6.1).

It turns out that if $G$ is strongly transitive on a thick building, then it inherits the structure of a $B N$ pair (also called a "Tits system"), cf. [1], Theorem 6.56. (A building is thick if, for each $s \in S$, each $s$-equivalence class contains at least three elements.)

1.3. Geometric realizations. A mirror structure over a set $S$ on a space $X$ is a family of subspaces $\left(X_{S}\right)_{s \in S}$ indexed by $S$. Given a mirror structure on $X$ and a subspace $Y \subset X$ there is an induced mirror structure on $Y$ defined by $Y_{s}:=Y \cap X_{s}$. If $X$ is a CW complex and each $X_{S}$ is a subcomplex, then $X$ is called a mirrored $C W$ complex. For each nonempty subset $T \subset S$, define subspaces $X_{T}$ and $X^{T}$ by

$$
X_{T}:=\bigcap_{s \in T} X_{s} \quad \text { and } \quad X^{T}:=\bigcup_{s \in T} X_{s} .
$$

Put $X_{\emptyset}:=X$ and $X^{\emptyset}:=\emptyset$. Given a cell $c$ of (a CW complex) $X$ or a point $x \in X$, put

$$
\begin{aligned}
& S(c):=\left\{s \in S \mid c \subset X_{s}\right\}, \\
& S(x):=\left\{s \in S \mid x \in X_{s}\right\} .
\end{aligned}
$$

Given a building $\mathcal{C}$ of type $(W, S)$ and a mirrored space $X$ over $S$, define an equivalence relation $\sim$ on $\mathcal{C} \times X$ by $(C, x) \sim(D, y)$ if and only if $x=y$ and $\delta(C, D) \in W_{S(x)}$ (i.e., $C$ and $D$ belong to the same $S(x)$-residue). The $X$-realization of $\mathcal{C}$, denoted $U(\mathcal{C}, X)$, is defined by

$$
u(\mathcal{C}, X):=(\mathcal{C} \times X) / \sim .
$$

( $e$ has the discrete topology.)

Example 1.5 (Spherical realizations). Suppose that $W$ is a finite Coxeter group of rank $n+1$ and $\mathcal{C}$ is a spherical building of type $(W, S)$. Then $W$ is a reflection 
group on the unit sphere $S^{n} \subset \mathbb{R}^{S}$. Let $\Delta^{n} \subset S^{n}$ be the fundamental simplex with its codimension one faces indexed by $S$. The space $\mathcal{U}\left(\mathcal{C}, \Delta^{n}\right)$ is called the spherical realization of $\mathcal{C}$. It is homotopy equivalent to a wedge of $n$-spheres (cf. [1], Theorem 4.127).

Geometric realizations of posets. Given a poset $\mathcal{T}, \operatorname{Flag}(\mathcal{T})$ denotes the set of finite chains in $\mathcal{T}$, partially ordered by inclusion, i.e., an element of $\operatorname{Flag}(\mathcal{T})$ is a finite, nonempty, totally ordered subset of $\mathcal{T}$. $\operatorname{Flag}(\mathcal{T})$ is an abstract simplicial complex with vertex set $\mathcal{T}$ and with $k$-simplices the elements of $\operatorname{Flag}(\mathcal{T})$ of cardinality $k+1$. The corresponding topological simplicial complex is the geometric realization of the poset $\mathcal{T}$ and is denoted by $|\mathcal{T}|$.

Suppose that $L$ is a simplicial complex with vertex set $S$. Let $S(L)$ be the poset of subsets of $S$ (including $\emptyset$ ) which are vertex sets of simplices in $L$. Let $K(L):=|S(L)|$ be the geometric realization of this poset. (Then $K(L)$ is isomorphic to the cone on the barycentric subdivision of $L$, where $\emptyset \in \delta$ provides the cone point.) Define a mirror structure on $K(L)$ by putting $K(L)_{s}:=\left|S(L)_{\geq\{s\}}\right|$.

Galleries in $\mathcal{C}$ and edge paths in $\boldsymbol{U}(\mathcal{C}, \boldsymbol{K}(\boldsymbol{L}))$. Let $v_{\emptyset}$ denote the central vertex of $K(L)$ corresponding to $\emptyset$ and for each $C \in \mathcal{C}$, let $v_{C}$ denote the image of $\left(C, v_{\emptyset}\right)$ in $\mathcal{U}(\mathcal{C}, K(L))$. The vertices $v_{C}$, with $C \in \mathcal{C}$, are the central vertices of $\mathcal{U}(\mathcal{C}, K(L))$. Let $v_{s}$ denote the vertex of $K(L)$ corresponding to $\{s\}$. If $\mathcal{R}$ is a residue of type $\{s\}$ (i.e., a "panel"), then for any $C \in \mathcal{R}$ the image of $\left(C, v_{s}\right)$ in $\mathcal{U}(\mathcal{C}, K(L))$ is denoted $v_{\mathcal{R}}$ and called a mirror vertex. The union of edges in $U(\mathcal{C}, K(L))$ connecting central vertices to mirror vertices is called the dual 1 -skeleton of $\mathcal{U}(\mathcal{C}, K(L))$. Given a gallery $\left(C_{0}, \ldots, C_{k}\right)$ in $C$ we get an edge path in the dual 1 -skeleton with successive vertices $\left(v_{C_{0}}, v_{\mathcal{R}_{1}}, v_{C_{1}}, \ldots, v_{C_{k}}\right)$. (There are $2 k+1$ vertices in this edge path; every other one has the form $v_{\mathcal{R}_{j}}$ where $\mathcal{R}_{j}$ is a residue of type $s_{j}$.) We will simplify notation by omitting the odd vertices and writing $\left(v_{C_{0}}, \ldots, v_{C_{k}}\right)$ for the corresponding edge path in $U(\mathcal{C}, K(L))$.

The standard realization, $\boldsymbol{U}(\mathcal{C}, \boldsymbol{K})$. As before, $\delta$ denotes the poset of spherical subsets of $S$. Put $K:=|\delta|$. For each $s \in S$, put $K_{s}:=\left|\delta_{\geq\{s\}}\right|$ and for each $T \in \mathcal{S}$, $K_{T}=\left|\delta_{\geq T}\right|$. The mirrored complex $K$ is sometimes called the Davis chamber of $(W, S)$ and $U(W, K)$, the Davis complex. The simplicial complex $U(\mathcal{C}, K)$ is the standard realization of $\mathcal{C}$. (Alternatively, $\mathcal{U}(\mathcal{C}, K)$ is the geometric realization of the poset of spherical residues of $\mathcal{C}$; see [11]). By construction $U(\mathcal{C}, K)$ is locally finite (since $\mathcal{C}$ is assumed to have finite thickness). It is proved in [11] that $U(\mathcal{C}, K)$ is contractible.

1.4. Duality groups. A space $X$ is an $n$-dimensional duality space if it is acyclic and if its compactly supported cohomology, $H_{c}^{*}(X)$, is torsion-free and concentrated in degree $n$. A discrete group $\Gamma$ is an $n$-dimensional duality group if $H^{*}(\Gamma ; \mathbb{Z} \Gamma)$ is 
torsion-free and concentrated in degree $n$. If a group $\Gamma$ acts freely and cocompactly on a duality space, then it is a duality group.

A finite simplicial complex $L$ has punctured (co)homology concentrated in degree $m$ if, for each closed simplex $\sigma$ of $L$ (including the empty simplex), the reduced cohomology, $\bar{H}^{*}(L-\sigma)$, is torsion-free and concentrated in degree $m$ (cf. [15], §6). We write $L$ is $\mathrm{PH}^{m}$ as a shorthand for this condition. For example, any triangulation of $S^{m}$ is $\mathrm{PH}^{m}$.

The following proposition is proved in [13] (also cf. [15], [16]).

Proposition 1.6 ([13], Corollary 9.5). Suppose that $\mathcal{C}$ is a building of type $(W, S)$. Then its standard realization, $U(\mathcal{C}, K)$, is an $n$-dimensional duality space if and only if the nerve, $L(W, S)$, is $\mathrm{PH}^{n-1}$.

\section{Constructing examples using covering spaces}

2.1. The main construction. Next we recall a construction of [10], §2. Suppose that $L$ is a simplicial complex with vertex set $S,\left(W^{\prime}, S^{\prime}\right)$ is a Coxeter system and $f: S \rightarrow S^{\prime}$ is a function defining a simplicial map (also denoted by $f$ ) from $L$ to the nerve $L\left(W^{\prime}, S^{\prime}\right)$ of $\left(W^{\prime}, S^{\prime}\right)$. Suppose also that the restriction of $f$ to each simplex is injective. (Most of the time, $f: S \rightarrow S^{\prime}$ will be a bijection and $W^{\prime}$ will be a finite Coxeter group.) Let $\left(m^{\prime}\left(s^{\prime}, t^{\prime}\right)\right)$ be the Coxeter matrix for $\left(W^{\prime}, S^{\prime}\right)$. Define an $(S \times S)$ Coxeter matrix $(m(s, t))$ by

$$
m(s, t):= \begin{cases}1 & \text { if } s=t, \\ m^{\prime}(f(s), f(t)) & \text { if }\{s, t\} \in \operatorname{Edge}(L), \\ \infty & \text { otherwise. }\end{cases}
$$

Let $(W, S)$ be the corresponding Coxeter system. The map $f: S \rightarrow S^{\prime}$ of generating sets extends to a homomorphism $\varphi_{f}: W \rightarrow W^{\prime}$. Let $\pi:=\operatorname{Ker}\left(\varphi_{f}\right)$. The space $K(L)$ is the cone on the barycentric subdivision of $L$ and as is explained in $\$ 1.3$, it has a mirror structure over $S$. Pushing forward via $f$, we get a mirror structure over $S^{\prime}$ on $K(L)$, defined by

$$
K(L)_{s^{\prime}}:=\bigcup_{s \in f^{-1}\left(s^{\prime}\right)} K(L)_{s} .
$$

As in $\$ 1.3$, we have the $W$-space, $U(W, K(L))$, and the $W^{\prime}$-space, $U\left(W^{\prime}, K(L)\right)$. The space $\mathcal{U}\left(W^{\prime}, K(L)\right)$ is connected if and only if $f: S \rightarrow S^{\prime}$ is surjective (cf. [10], Remark 2.2). Henceforth, assume this. Then $\varphi_{f}: W \rightarrow W^{\prime}$ is an epimorphism and it induces $\varphi_{f}$-equivariant map $q: U(W, K(L)) \rightarrow U\left(W^{\prime}, K(L)\right)$. It is easy to see that $q$ is a covering projection and that $W$ is the group of all lifts of the $W^{\prime}$-action. By [12], Theorem 9.1 .3 , p. $166, \mathcal{U}(W, K(L))$ is simply connected. Hence,

$$
\pi=\pi_{1}\left(U\left(W^{\prime}, K(L)\right)\right) \text {. }
$$


Remark. The space $\mathcal{U}(W, K(L))$ need not be contractible. A necessary and sufficient condition for this to be true is that for every spherical subset $T \subset S, T$ is the vertex set of a simplex in $L$, i.e., that $L=L(W, S)$.

Next we want to carry out the same construction for buildings. Suppose that we are given the same data as above, as well as a building $\ell^{\prime}$ of type $\left(W^{\prime}, S^{\prime}\right)$. Let $v_{\emptyset}$ be the central vertex of $K(L)$ corresponding to $\emptyset$. For each $C^{\prime} \in \mathcal{C}^{\prime}$, let $v_{C^{\prime}}$ denote the corresponding central vertex of $\mathcal{U}\left(\mathcal{C}^{\prime}, K(L)\right)$. We can identify $\ell^{\prime}$ with the set $\operatorname{Cent}\left(U\left(\mathcal{C}^{\prime}, K(L)\right)\right):=\left\{v_{C^{\prime}}\right\}_{C^{\prime} \in \mathcal{C}^{\prime}}$ of central vertices. By analogy with the case of Coxeter groups, consider the universal cover $p: \widetilde{U} \rightarrow U\left(\mathcal{C}^{\prime}, K(L)\right)$. Define

$$
\ell:=\operatorname{Cent}(\tilde{u}):=p^{-1}\left(\operatorname{Cent}\left(u\left(\ell^{\prime}, K(L)\right)\right) .\right.
$$

Let $\tilde{\pi}$ denote the fundamental group of $U\left(\ell^{\prime}, K(L)\right)$. There is a free action of $\tilde{\pi}$ on $\zeta$ such that the quotient set is identified with $\ell^{\prime}$. Since $K(L)$ is simply connected (it is a cone), $p^{-1}(K(L))$ is isomorphic to $\tilde{\pi} \times K(L)$. For each $C \in \mathcal{C}$, let $C_{\langle K\rangle}$ denote the component of $p^{-1}(K(L))$ which contains the vertex $C$. We shall say that $C_{\langle K\rangle}$ is a chamber of $\tilde{u}$. Note that $\left.p\right|_{C_{\langle K\rangle}}$ maps $C_{\langle K\rangle}$ homeomorphically onto $K(L)$.

We want to show that $\mathcal{C}$ has the structure of a building of type $(W, S)$ and that $\tilde{u} \cong U(\mathcal{C}, K(L))$. To this end, we first show $\mathcal{C}$ has the structure of a chamber system over $S$. Chambers $C, D \in \mathcal{C}$ are s-adjacent if $C_{\langle K\rangle} \cap D_{\langle K\rangle}$ is nonempty and projects homeomorphically onto $K(L)_{f(s)}$. A gallery in $\mathcal{C}(=\operatorname{Cent}(\tilde{\mathcal{U}}))$ is reduced if its type $\boldsymbol{s}$ is a reduced expression for $w(\boldsymbol{s})$.

Lemma 2.1. Let $\mathcal{C}=\operatorname{Cent}(\tilde{U})$ be defined by (2). Suppose that $\left(C_{0}, \ldots, C_{k}\right)$ is a gallery in $\tilde{U}$ of type $\boldsymbol{s}$ and that $\boldsymbol{s}^{\prime}$ is another word in $S$ which can be obtained from $\boldsymbol{s}$ by flip moves. Then there is a gallery in $\tilde{U}$ of type $\boldsymbol{s}^{\prime}$ with the same endpoints.

Proof. Suppose that $T \in S(W, S)$ is a spherical subset. Then the restriction of $p$ to a $T$-residue in $\mathcal{C}$ is a bijection to (the set of central vertices in) a $T$-residue of $\mathcal{C}^{\prime}$. Hence, every $T$-residue in $\mathcal{C}$ is a spherical building. It suffices to show that we can accomplish a single flip move on $s$ without changing the endpoints. So, suppose that $\boldsymbol{s}=\operatorname{prod}(s, t ; m(s, t))$, where $m(s, t)<\infty$. Then the entire gallery from $C_{0}$ to $C_{k}$ (where $k=m(s, t))$ lies in a single $\{s, t\}$-residue. Since this residue is a building and $\operatorname{prod}(s, t ; m(s, t))$ is a reduced expression, there is a gallery of type $\operatorname{prod}(t, s ; m(s, t))$ with the same endpoints. In the general case we apply this step to the subgallery in which the flip is supposed to take place, proving the lemma.

Tits' solution to the word problem for Coxeter groups gives the following.

Corollary 2.2. Suppose that $\left(C_{0}, \ldots, C_{k}\right)$ is a gallery in $\tilde{U}$ of type $s$ with $s$ a reduced expression for an element of $W$. Let $\boldsymbol{s}^{\prime}$ be another reduced expression for $w(\boldsymbol{s})$. Then there is a gallery of type $\boldsymbol{s}^{\prime}$ from $C_{0}$ to $C_{k}$. 
Lemma 2.3. Suppose that $\boldsymbol{C}=\left(C_{0}, \ldots, C_{k}\right)$ is a gallery of type $\mathbf{s}$ from $C_{0}$ to $C_{k}$. If $\boldsymbol{C}$ has minimum length, then $\boldsymbol{s}$ is a reduced expression.

Proof. If $\boldsymbol{s}$ is not reduced, then, by Tits' solution to the word problem, $\boldsymbol{s}$ can be changed by a sequence of flips to $\boldsymbol{s}^{\prime}=\left(s_{1}^{\prime}, \ldots, s_{k}^{\prime}\right)$ so that $\boldsymbol{s}^{\prime}$ contains a subword of length two of the form $\left(s_{j}^{\prime}, s_{j+1}^{\prime}\right)=(s, s)$. By Lemma 2.1 , there is a gallery $\boldsymbol{C}^{\prime}=\left(C_{0}^{\prime}, \ldots, C_{k}^{\prime}\right)$ with the same endpoints as $C$. Let $\left(C_{j-1}^{\prime}, C_{j}^{\prime}, C_{j+1}^{\prime}\right)$ be the subgallery corresponding to the subword $(s, s)$. Then $C_{j-1}^{\prime}, C_{j}^{\prime}$ and $C_{j+1}^{\prime}$ are all $s$-equivalent. If $C_{j+1}^{\prime}=C_{j-1}^{\prime}$, then we can shorten the gallery by deleting $C_{j-1}^{\prime}$ and $C_{j}^{\prime}$. If not, we can shorten it by deleting $C_{j}^{\prime}$. In either case we contradict the assumption that $C$ has minimum length.

Next, we want to define the Weyl distance $\delta: \mathcal{C} \times \ell \rightarrow W$. Fix a chamber $C^{\prime} \in \mathcal{C}^{\prime}$ and let $\rho_{C^{\prime}}: \mathcal{C}^{\prime} \rightarrow W^{\prime}$ be the function $D^{\prime} \mapsto \delta^{\prime}\left(C^{\prime}, D^{\prime}\right)$. Since $\rho_{C^{\prime}}$ maps $s^{\prime}$-equivalent chambers to $s^{\prime}$-equivalent elements of $W^{\prime}$, it induces a map of spaces $r_{C^{\prime}}: U\left(\mathcal{C}^{\prime}, K(L)\right) \rightarrow \mathcal{U}\left(W^{\prime}, K(L)\right)$, which can be thought of as the realization of retraction onto an apartment. Choose a chamber $C \in \mathcal{C}$ lying over $C^{\prime}$. We want to construct a map $r_{C}: \tilde{U} \rightarrow \mathcal{U}(W, K(L))$ making the following diagram commute:

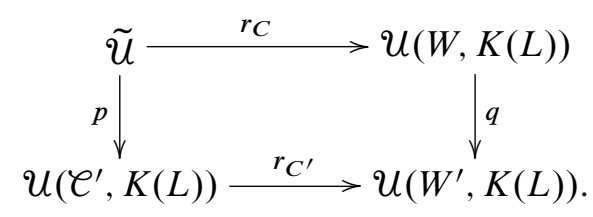

Since $q$ is a covering projection and $U(W, K(L))$ is simply connected, the standard lifting theorem in covering space theory implies that $r_{C^{\prime}} \circ p: \tilde{u} \rightarrow U\left(W^{\prime}, K(L)\right)$ has a unique lift $r_{C}$, as in the diagram, taking the central vertex $C$ to 1 . Restricting $r_{C}$ to the subset $\ell \subset \tilde{U}$, we get a function $\rho_{C}: \ell \rightarrow W$. (Recall that $\ell$ is the set of central vertices in $\tilde{U}$ and $W$ is the set of central vertices in $U(W, K(L))$.) Define $\delta: \mathcal{e} \times \boldsymbol{\ell} \rightarrow W$ by

$$
\delta(C, D):=\rho_{C}(D) .
$$

Remark. The reason this procedure works comes down to a fact, which was used in the proof of Lemma 2.1: every spherical residue in the chamber system $\ell$ is a spherical building. This together with simple connectivity of the standard realization implies that $\mathcal{C}$ is a building (cf. Tits [28]).

Lemma 2.4. Suppose that $\boldsymbol{C}=\left(C_{0}, \ldots, C_{k}\right)$ is a reduced gallery in $\mathcal{C}$ of type $\boldsymbol{s}$. Then $\delta\left(C_{0}, C_{k}\right)=w(s)$.

Proof. Let $\gamma:[0, k] \rightarrow \tilde{u}$ be the edge path in the dual 1-skeleton of $\tilde{\mathcal{U}}$ corresponding to $C$. Thus, $\gamma$ crosses the central vertices $C_{0}, C_{1} \ldots, C_{k}$ in succession. Then $r_{C_{0}} \circ \gamma$ 
is an edge path in the dual 1-skeleton of $\mathcal{U}(W, K(L))$. Let $\left(w_{0}, w_{1}, \ldots, w_{k}\right)$ be the corresponding gallery in $W$. By construction the type of this gallery is $\boldsymbol{s}$ (the type of $\boldsymbol{C})$. Since $r_{C_{0}}\left(C_{0}\right)=1$, the initial vertex $w_{0}$ is 1 . Thus, $\delta\left(C_{0}, C_{k}\right)=r_{C_{0}}\left(C_{k}\right)=$ $w_{k}=w(s)$.

Theorem 2.5. The map $\delta: \mathcal{C} \times \mathcal{C} W W$ is a Weyl distance function; hence, $\mathcal{C}$ is a building of type $(W, S)$. Moreover, $\tilde{U}$ can be identified with $\mathcal{U}(\mathcal{C}, K(L))$.

Proof. We must check conditions (WD1), (WD2), (WD3) of Definition 1.2. The first condition is immediate. To check (WD2) suppose that $\delta(C, D)=w$ and $C^{\prime} \in \mathcal{C}$ satisfies $\delta\left(C^{\prime}, C\right)=s$. By definition of $\delta$ there is a gallery $\left(C_{0}, \ldots, C_{k}\right)$ of type $s$ from $C$ to $D$, where $s=\left(s_{1}, \ldots, s_{k}\right)$ is a reduced expression for $w$. There are two cases to consider.

Case 1: $l(s w)>l(w)$. Then $\boldsymbol{s}^{\prime}=\left(s, s_{1}, \ldots, s_{k}\right)$ is a reduced expression for $s w$ and $\left(C^{\prime}, C_{0}, \ldots, C_{k}\right)$ is a reduced gallery of type $s^{\prime}$. So, by Lemma $2.4, \delta\left(C^{\prime}, C_{k}\right)=$ $w\left(s^{\prime}\right)=s w$.

Case 2: $l(s w)<l(w)$. Then there is a reduced expression $\boldsymbol{s}^{\prime}$ for $w$ which begins with $s$. By Corollary 2.2, there is a gallery of type $s^{\prime}$ from $C$ to $D$. The resulting gallery $\left(C^{\prime}, C, C_{1}, \ldots, D\right)$ has type beginning $(s, s)$. Hence, the chambers $C^{\prime}, C$ and $C_{1}$ are $s$-equivalent. If $C_{1}=C^{\prime}$, then $\left(C_{1}, C_{2}, \ldots\right)$ is a reduced gallery from $C^{\prime}$ to $D$; hence, $\delta\left(C^{\prime}, D\right)=s w$. If $C_{1} \neq C$, then $\left(C^{\prime}, C_{1}, C_{2}, \ldots\right)$ has type $s^{\prime}$ and is a gallery from $C^{\prime}$ to $D$. Hence, $\delta\left(C^{\prime}, D\right)=w\left(\boldsymbol{s}^{\prime}\right)=w$.

To check (WD3), let $s$ be an arbitrary element of $S$. Suppose that we have a reduced gallery $\left(C_{0}, \ldots, C_{k}\right)$ from $C$ to $D$ of type $\left(s_{1}, \ldots, s_{k}\right)$. The argument divides into the same two cases as above. In Case $1,\left(C^{\prime}, C_{0}, C_{1}, \ldots, C_{k}\right)$ is a reduced gallery; hence, $\delta\left(C^{\prime}, C_{k}\right)=s w$. In Case 2, by using Corollary 2.2 and Lemma 2.4, we can assume that $s_{1}=s$ (i.e., the type of the gallery begins with $s$ ). Then choosing $C^{\prime}=C_{1}$ we get a reduced gallery $\left(C_{1}, \ldots, C_{k}\right)$ from $C^{\prime}$ to $D$; hence, $\delta\left(C^{\prime}, D\right)=s w$.

Examples 2.6. Suppose that $W^{\prime}$ is a finite Coxeter group of rank $n+1$ with generators indexed by $S^{\prime}=\{0,1, \ldots, n\}$. Then $L^{\prime}\left(=L\left(W^{\prime}, S^{\prime}\right)\right)$ is the simplex on $\{0,1, \ldots, n\}$. Suppose that $L$ is an $n$-dimensional simplicial complex which admits a "folding map" $f: L \rightarrow L^{\prime}$ (i.e., the restriction of $f$ to each simplex is injective). Then we can proceed as above. In particular:

(1) Suppose that $L$ is the barycentric subdivision of an $n$-dimensional cell complex. Then there is a folding map $f: S \rightarrow S^{\prime}$ which sends the barycenter of a cell $F$ to $\operatorname{dim} F$. If $L$ is the barycentric subdivision of the boundary complex of an $(n+1)$-dimensional polytope, then $K(L)$ is a simple polytope. For example, if $L$ is the boundary of a $(n+1)$-simplex, then $K(L)$ is an $(n+1)$-dimensional permutohedron (cf. Remark 6.6 in [10]). In the building $U(\mathcal{C}, K(L))$ the link of each vertex of $K(L)$ is the spherical building $U\left(\mathcal{C}^{\prime}, \Delta^{n}\right)$, where $\Delta^{n}$ is the dual simplex to $L^{\prime}$. 
(2) Suppose that $L$ is the boundary complex of an $(n+1)$-dimensional octahedron (see $\$ 3.2$ below). Its vertex set, $S$, can be identified with $\{0, \ldots, n\} \times\{ \pm 1\}$ and the folding map is induced by projection on the first factor, $\{0, \ldots, n\} \times\{ \pm 1\} \rightarrow$ $\{0, \ldots, n\}$. In this case, $K(L)$ is an $(n+1)$-cube. As in (1), the link of each vertex of $K(L)$ in $\mathcal{U}(\mathcal{C}, K(L))$ is the spherical building $U\left(\mathcal{C}^{\prime}, \Delta^{n}\right)$.

Definition 2.7. A simplicial complex $J$ is a flag complex if any nonempty, finite set of vertices, which are pairwise connected by edges, spans a simplex of $J$.

A flag complex $J$ is "determined by its 1-skeleton" in the sense that it is the smallest full subcomplex of the simplex on $\operatorname{Vertex}(J)$ which contains the 1-skeleton $J^{1}$.

Theorem 2.8. If in the above construction $L$ is a flag complex, then $L=L(W, S)$ (and hence, $U(\mathcal{C}, K(L))$ is the standard realization of $\mathcal{C}$ ).

Proof. Suppose that $T$ is a spherical subset of $S$. Since this implies that $m(s, t)<\infty$ for all $s, t \in T$, we see that the elements of $T$ are pairwise connected by edges in $L$. Since $L$ is a flag complex, this means that $T$ is the vertex set of a simplex in $L$. Hence, $L(W, S)=L$.

Remark. When $W^{\prime}$ is finite, the condition that $L$ be a flag complex is also necessary for $L$ to equal $L(W, S)$.

Example 2.9. Given $\left(W^{\prime}, S^{\prime}\right)$, choose a set of edges $E \subset \operatorname{Edge}\left(L\left(W^{\prime}, S^{\prime}\right)\right)$. Let $f: S \rightarrow S^{\prime}$ be any bijection. As in $(1)$, define a Coxeter matrix $(m(s, t))$ by

$$
m(s, t)= \begin{cases}m^{\prime}(f(s), f(t)) & \text { if }\{s, t\} \notin E, \\ \infty & \text { if }\{s, t\} \in E .\end{cases}
$$

Let $(W, S)$ be the resulting Coxeter system and $L=L(W, S)$. So, for any building $\ell^{\prime}$ of type $\left(W^{\prime}, S^{\prime}\right)$, we get a building $\mathcal{C}$ of type $(W, S)$.

Here is slightly different spin on the same example. Suppose that $L$ is an arbitrary finite flag complex with vertex set $S$. Then $L$ is a subcomplex of the simplex $\Delta$ on $S$. Let $\left(W^{\prime}, S\right)$ be an arbitrary spherical Coxeter system. Then $L\left(W^{\prime}, S\right)=\Delta$. If $E$ is the set of edges of $\Delta$ which are not in $L$, then the construction in the previous paragraph yields $(W, S)$ with $L(W, S)=L$. For $L \neq \Delta$, we could have reached the same conclusion by choosing $\left(W^{\prime}, S\right)$ to be any Coxeter system with nerve $\partial \Delta$. For example, $\left(W^{\prime}, S^{\prime}\right)$ could be an irreducible affine Coxeter system.

Suppose that the flag complex $L$ is $\mathrm{PH}^{n-1}$ (defined in $\S 1.4$ ). Then, by Proposition 1.6, $U(\mathcal{C}, K(L))$ is an $n$-dimensional duality space and, by Corollary 2.12 below, $\operatorname{Aut}(\mathcal{C})$ is an $n$-dimensional duality group. 


\subsection{Automorphism groups}

Proposition 2.10. Suppose that $G^{\prime}$ is a group of automorphisms of $\mathcal{C}^{\prime}$. Then $\mathcal{C}$ has a group of automorphisms $G$ with $\mathcal{\ell} / G \cong \mathcal{C}^{\prime} / G^{\prime}$ and $U(\mathcal{C}, K(L)) / G \cong$ $u\left(\mathcal{C}^{\prime}, K(L)\right) / G^{\prime}$.

Proof. By Theorem 2.5, $U(\mathcal{C}, K(L)) \rightarrow U\left(\mathcal{C}^{\prime}, K(L)\right)$ is the universal covering. Let $G$ be the group of all lifts of the $G^{\prime}$-action. The asserted isomorphisms are easily established.

Corollary 2.11. If $\ell^{\prime}$ admits a chamber-transitive group of automorphisms, then so does $\mathcal{C}$. More generally, if the action of a group $G^{\prime}$ of automorphisms of $\mathcal{C}^{\prime}$ has only finitely many orbits, then the G-action on $C$ has the same number of orbits.

Corollary 2.12. If $\mathcal{C}^{\prime}$ is a spherical building, then Aut $(\mathcal{C})$ admits a torsion-free uniform lattice.

Proof. The discrete subgroup $\tilde{\pi} \subset \operatorname{Aut}(\mathcal{C})$ is such a lattice (with $\tilde{\pi}:=\pi_{1}\left(U\left(\mathcal{C}^{\prime}, K\right)\right.$ ). It is uniform, since $\ell / \tilde{\pi}=\ell^{\prime}$, which is a finite set. It is torsion-free, since it acts freely on the finite dimensional, contractible space $U(\mathcal{C}, K)$.

Question. When is $\operatorname{Aut}(\mathcal{C})$ strongly transitive?

In the cases of classical or Kac-Moody buildings, Aut( $(\mathcal{C})$ is usually strongly transitive. It is also strongly transitive in the case of a right-angled building which is regular and thick, cf. Bourdon [5] and Barnhill-Thomas [4]. Very little is known in other cases.

\section{Partial products of Coxeter groups and buildings}

3.1. General description. Suppose that we are given as data:

- Coxeter systems $\left(W_{i}, S_{i}\right)$, for $i=0,1, \ldots, p$;

- for each pair $(i, j)$ with $0 \leq i, j \leq p$ and $i \neq j$, a subset $\mathcal{R}_{i j} \subset S_{i} \times S_{j}$ such that $\mathcal{R}_{j i}$ is obtained from $\mathcal{R}_{i j}$ simply by interchanging the factors (i.e., $\left.\left(s_{j}, s_{i}\right) \in \mathcal{R}_{j i} \Longleftrightarrow\left(s_{i}, s_{j}\right) \in \mathcal{R}_{i j}\right)$.

From this data we define a new Coxeter system $(W, S)$. First, $S$ is the disjoint union $S:=S_{0} \amalg \ldots \amalg S_{p}$. For $s_{i}, t_{i} \in S_{i}$, let $m_{i}\left(s_{i}, t_{i}\right)$ denote the order of $s_{i} t_{i}$. Define an $(S \times S)$ symmetric matrix $(m(s, t))$ as follows: given $s \in S_{i}, t \in S_{j}$,

$$
m(s, t)= \begin{cases}m_{i}(s, t) & \text { if } i=j, \\ \infty & \text { if } i \neq j \text { and }(s, t) \in \mathcal{R}_{i j}, \\ 2 & \text { if } i \neq j \text { and }(s, t) \notin \mathcal{R}_{i j} .\end{cases}
$$


Define $W$ to be the Coxeter group associated to the Coxeter matrix $(m(s, t))$. In other words, the $\mathcal{R}_{i j}$ specify the edges from a vertex in $S_{i}$ to one in $S_{j}$ which are to be deleted from the nerve of $\left(W_{0} \times \cdots \times W_{p}, S\right)$.

Examples 3.1. (1) Suppose that $\mathcal{R}_{i j}=\emptyset$ for all $i \neq j$. Then $W$ is the direct product $W_{0} \times W_{1} \times \cdots \times W_{p}$.

(2) Suppose that $\mathcal{R}_{i j}=S_{i} \times S_{j}$ for all $i \neq j$. Then $W$ is the free product $W_{0} * W_{1} * \cdots * W_{p}$.

(3) Suppose that $\Omega$ is a simplicial graph with vertex set $\{0,1, \ldots, p\}$ and

$$
\mathcal{R}_{i j}= \begin{cases}\emptyset & \text { if }\{i, j\} \in \operatorname{Edge}(\Omega), \\ S_{i} \times S_{j} & \text { if }\{i, j\} \notin \operatorname{Edge}(\Omega) .\end{cases}
$$

Then $W$ is the "graph product" of the $W_{i}$, denoted by $\prod_{\Omega} W_{i}$. (See [22] for discussion of graph products.) As a further specialization, if each $W_{i}$ is cyclic of order two, then $W=\prod_{\Omega} \mathbb{Z} / 2$ is the right-angled Coxeter group associated to $\Omega$. If each $W_{i}$ is the infinite dihedral group, $D_{\infty}$, then $W=\prod_{\Omega} D_{\infty}$ is commensurable with the right-angled Artin group associated to $\Omega$ (cf. [14]).

Example 3.2 (Square products). In this example suppose that the sets $S_{i}$ are mutually bijective. Assume further that we are given a compatible family of bijections, $\left\{\theta_{i j}: S_{i} \rightarrow S_{j}\right\}_{0 \leq i, j \leq p}$. (By a "compatible family" we mean that $\theta_{i i}=1$ and $\theta_{j k} \circ \theta_{i j}=\theta_{i k}$, for all $i, j, k$.) The $\mathcal{R}_{i j}$ are required to satisfy the following: the bijection $\theta_{i 0} \times \theta_{j 0}: S_{i} \times S_{j} \rightarrow S_{0} \times S_{0}$ takes $\mathcal{R}_{i j}$ onto the diagonal of $S_{0} \times S_{0}$ (or equivalently, $\left(\theta_{i j} \times \mathrm{id}\right)\left(\mathcal{R}_{i j}\right)$ is the diagonal of $\left.S_{j} \times S_{j}\right)$. Then for $s \in S_{i}, t \in S_{j}$,

$$
m(s, t)= \begin{cases}m_{i}(s, t), & \text { if } i=j, \\ \infty, & \text { if } i \neq j \text { and } \theta_{i j}(s)=t, \\ 2, & \text { otherwise. }\end{cases}
$$

In this case call $W$ the square product of the $W_{i}$ and write $W=W_{0} \square \cdots \square W_{p}$. (In the case where there are only two factors and $W_{0}$ and $W_{1}$ are both finite, these examples were discussed in Example 4.3 in [10].)

Spherical subsets of $S$. Let $S_{i}:=\delta\left(W_{i}, S_{i}\right)$ be the poset of spherical subsets of $S_{i}$ and let $S:=S(W, S)$ be the poset of spherical subsets of $S$. Any $T \subset S$ can be decomposed as $T=T_{0} \amalg \cdots \amalg T_{p}$, where $T_{i} \subset S_{i}$. Clearly, $T$ is spherical if and only if the following two conditions hold:

- $T_{i} \in \mathcal{S}_{i}$, for $i=0, \ldots, p$, and

- $\left(s_{i}, s_{j}\right) \notin \mathcal{R}_{i j}$, for all $s_{i} \in T_{i}, s_{j} \in T_{j}, i \neq j$. 
Put

$$
W^{\prime}:=W_{0} \times \cdots \times W_{p} .
$$

Note that $\delta\left(W^{\prime}, S\right)=\S_{0} \times \cdots \times \delta_{p}$. As in $\S 2$, the tautological bijection $f: S \rightarrow$ $S_{0} \amalg \cdots \amalg S_{p}$ extends to an epimorphism $\varphi_{f}: W \rightarrow W^{\prime}$ and induces an embedding $\delta \rightarrow \delta\left(W^{\prime}, S\right)$ defined by $T \mapsto\left(T_{0}, \ldots, T_{p}\right)$ (where $\left.T=T_{0} \amalg \cdots \amalg T_{p}\right)$. Similarly, if $L:=L(W, S)$ and $L_{i}:=L\left(W_{i}, S_{i}\right)$ denote the nerves of their respective Coxeter systems, we get a simplicial embedding $f: L \rightarrow L\left(W^{\prime}, S\right)$. (Note that $L\left(W^{\prime}, S\right)$ is the join, $L_{0} * \cdots * L_{p}$.)

Buildings. For $0 \leq i \leq p$, suppose that $\mathcal{C}_{i}$ is a building of type $\left(W_{i}, S_{i}\right)$. Then $\ell_{0} \times \cdots \times \ell_{p}$ is a building of type $\left(W^{\prime}, S\right)$. As in $\$ 2$ this lead to a new building $\ell$ and a covering space, $\mathcal{U}(\mathcal{C}, K) \rightarrow \mathcal{U}\left(\mathcal{C}^{\prime}, K\right)$ where $K:=K(L)$ is the fundamental chamber for $(W, S)$.

If $W$ is as in Examples 3.1 part (1), (2) or (3), then $\mathcal{C}$ is, respectively, the product, free product or graph product of the $\boldsymbol{C}_{i}$. In the case of the graph product, write $\ell=\prod_{\Omega} \ell_{i}$. If $W$ is as in Example 3.2, then $\mathcal{C}=\ell_{0} \square \cdots \square \ell_{p}$ is the square product of the $\mathcal{C}_{i}$.

Example 3.3 (The free product of two buildings). Suppose that $\ell_{0}$ and $\ell_{1}$ are buildings of type $\left(W_{0}, S_{0}\right)$ and $\left(W_{1}, S_{1}\right)$, respectively, and that for $i=0,1, K(i):=$ $K\left(W_{i}, S_{i}\right)$. Let $K:=K(0) \vee K(1)$ denote the one point union at the central vertex. Then $K$ naturally has a mirror structure over $S_{0} \amalg S_{1}$. By definition, the free product, $\ell_{0} * \ell_{1}$, is the set of chambers in the universal cover of $U\left(\ell_{0} \times \ell_{1}, K\right)$. It is a building of type $\left(W_{0} * W_{1}, S_{0} \amalg S_{1}\right)$. For example, suppose that each $\ell_{i}$ is the edge set of a tree $T_{i}$ (without terminal vertices). A fundamental chamber for $T_{0} \times T_{1}$ has the form $e_{0} \times e_{1}$ where $e_{i}$ is an edge of $T_{i}$. The space $K$ is defined to be the subspace $\left(m_{0} \times e_{1}\right) \cup\left(e_{0} \times m_{1}\right)$, where $m_{i}$ denotes the midpoint of $e_{i}$. (In other words, $K$ is the wedge of two edges at their midpoints.) The space $\left.T_{0} \times T_{1}=U\left(\ell_{0} \times \ell_{1}, e_{0} \times e_{1}\right)\right)$ is a 2-dimensional complex tiled by squares. To get $U\left(\ell_{0} \times \ell_{1}, K\right)$ remove from each square everything except $K$. The resulting 1-dimensional complex is not simply connected. Its universal cover is by definition $U\left(\ell_{0} * \ell_{1}, K\right)$.

We have the following corollary to Corollary 2.12 .

Corollary 3.4. Suppose that each $\ell_{i}$ is a spherical building (of finite thickness) and that $\Omega$ is a simplicial graph. If $\Omega$ is not the 1-skeleton of a simplex, then the graph product $\prod_{\Omega} \mathcal{C}_{i}$ is an infinite building whose automorphism group admits a uniform lattice. Similarly, if $p \geq 1$, then $\mathcal{C}_{0} \square \cdots \square \mathcal{C}_{p}$ is an infinite building whose automorphism group admits a uniform lattice. 
3.2. Description of $\boldsymbol{L}$ for $\square$-products. Suppose that $V$ is a set with $n$ elements and $\Delta(V)$ is the simplex on $V$. Define a mirror structure over $V$ on $\Delta(V)$ by letting $\Delta(V)_{v}$ be the codimension one face opposite to the vertex $v$. Let $(\mathbb{Z} / 2)^{V}$ be the direct product of $V$ copies of the cyclic group of order two. Identify $V$ with the standard basis of $(\mathbb{Z} / 2)^{V}$ (so that $\left((\mathbb{Z} / 2)^{V}, V\right)$ is a Coxeter system). Then $U\left((\mathbb{Z} / 2)^{V}, \Delta(V)\right.$ ) is a triangulation of $S^{n-1}$, specifically, it is the boundary complex of an $n$-dimensional octahedron.

For any integer $p \geq 1$, the set $\{0, \ldots, p\}^{V}$ is a building of type $\left((\mathbb{Z} / 2)^{V}, V\right)$ and thickness $p$. Its realization $U\left(\{0, \ldots, p\}^{V}, \Delta(V)\right)$ is an $n$-dimensional spherical building. The space $U\left(\{0, \ldots, p\}^{V}, \Delta(V)\right)$ is a simplicial complex which we denote $O(p, V)$. The simplices of $O(p, V)$ can be described as follows. Consider pairs of the form $g \times \Delta(U)$ where $U \subset V$ and $g=\left(g_{v}\right)_{v \in V}$ is a $V$-tuple in $\{0, \ldots, p\}$. Two such pairs $g \times \Delta(U)$ and $g^{\prime} \times \Delta\left(U^{\prime}\right)$ are equivalent if and only $U=U^{\prime}$ and $g_{v}=g_{v}^{\prime}$ for all $v \in U$. A simplex in $O(p, V)$ is an equivalence class.

The complex $O(p, V)$ can also be described as the $n$-fold join of the set $\{0, \ldots, p\}$ with itself.

\section{Lemma 3.5. $O(p, V)$ is $\mathrm{PH}^{n-1}$.}

Proof. The vertex set of an $(m-1)$-simplex $\sigma$ in $O(p, V)$ is a $U$-tuple $g \in\{0, \ldots, p\}^{U}$, for some $U$ with $m=\operatorname{Card}(U)$. The complement of $\sigma$ in $O(p, V)$ deformation retracts onto the complement of the open star of $\sigma$. The complement of this open star can be identified with the join of $m$ sets of $p$ elements and $n-m$ sets of $p+1$ elements. It is well-known that the join of $n$ finite sets, each of which has at least 2 elements, is homotopy equivalent to a wedge of $(n-1)$-spheres. On the other hand, if at least one of the sets in an $n$-fold join has exactly one point, then the join is contractible. The lemma follows.

As before, $\left(W_{i}, S_{i}\right)$ are Coxeter systems, $\theta_{i j}: S_{i} \rightarrow S_{j}$ are compatible bijections, $L_{i}=L\left(W_{i}, S_{i}\right)$ and $S_{i}=\delta\left(W_{i}, S_{i}\right)$. Also, $S:=S_{0} \amalg \cdots \amalg S_{p}$ and $W:=$ $W_{0} \square \cdots \square W_{p}$. Put $V:=S_{0}$. Using the bijections $\theta_{i 0}: S_{i} \rightarrow S_{0}$ identify each $S_{i}$ with $V$. We are going to define an embedding of posets $S \hookrightarrow S(O(p, V))$. The image will be denoted $S_{0} \square \cdots \square S_{p}$. An element of $S$ has the form $\left(T_{0}, \ldots, T_{p}\right)$, where $T_{i} \subset S_{i}$. Use the $\theta_{i 0}$ to transport the $T_{i}$ to subsets $U_{i}$ of $V$, i.e., put $U_{i}:=\theta_{i 0}\left(T_{i}\right)$. The fact that each $\mathcal{R}_{i j}$ is the diagonal of $V \times V$ means that the $U_{i}$ are pairwise disjoint subsets of $V$. Put $U:=U_{0} \cup \cdots \cup U_{p}$. Since the $U_{i}$ are disjoint, any $u \in U$ belongs to exactly one of them, call it $U_{g_{u}}$. This defines a $U$-tuple $g \in\{0, \ldots, p\}^{U}$. Extend this arbitrarily to a $V$-tuple in $\{0, \ldots, p\}$ and also denote it by $g$. (In other words, $g_{v}$ is completely arbitrary for $v \in V-U$.) The embedding $S \hookrightarrow S(O(p, V))$ is then defined by $\left(T_{0}, \ldots, T_{p}\right) \mapsto(U, g)$. The corresponding subcomplex of $O(p, V)$ is denoted $L_{0} \square \cdots \square L_{p}$. This discussion proves the following. 
Proposition 3.6. With notation as above,

$$
L(W, S) \cong L_{0} \square \cdots \square L_{p} \subset O(p, V) .
$$

Remark. The above description of $L=L_{0} \square \cdots \square L_{p}$ gives the following estimate for its dimension:

$$
\max _{i}\left\{\operatorname{dim} L_{i}\right\} \leq \operatorname{dim} L \leq n-1
$$

Corollary 3.7. Suppose that each $W_{i}$ is a finite Coxeter group of rank $n$ and $V$ is a set with $n$ elements. Then $L(W, S) \cong O(p, V)$.

Combining this with Lemma 3.5 and Proposition 1.6 we get the following.

Corollary 3.8. Suppose that each $W_{i}$ is a finite Coxeter group of rank $n$ so that each $\mathcal{C}_{i}$ is a spherical building. Then the standard realization of $\bigodot_{0} \square \cdots \square \bigodot_{p}$ is an $n$-dimensional duality space and $\operatorname{Aut}\left(\mathcal{C}_{0} \square \cdots \square \mathcal{C}_{p}\right)$ is a virtual n-dimensional duality group.

Example 3.9. When $p=1, O(1, V)$ is the boundary complex of an $n$-dimensional octahedron. So, in this case, $K(=K(L))$ is combinatorially isomorphic to an $n$-cube. Suppose that $W_{0}, W_{1}$ are finite Coxeter groups of rank $n$. Then $K$ is the $n$-cube and in the standard realization, $\mathcal{U}\left(W_{0} \square W_{1}, K\right)$, the isotropy group at one vertex of $K$ is $W_{0}$ while at the opposite vertex it is $W_{1}$, cf. [10], Example 4.3. It follows that $W_{0} K$ is combinatorially isomorphic to a Coxeter cell of type $\left(W_{0}, S_{0}\right)$. For example, if $W_{0}$ is the symmetric group of rank $n+1$, then $W_{0} K$ is a permutohedron, cf. [10], §6. Next suppose that, for $i=0,1, \mathcal{C}_{i}$ is a spherical building of type $\left(W_{i}, S_{i}\right)$. In the standard realization, $U\left(\ell_{0} \square \ell_{1}\right)$, the link at one vertex is the spherical realization $U\left(\ell_{0}, \Delta\right)$ while at the other it is $U\left(\mathcal{C}_{1}, \Delta\right)$. At the other vertices, the links are joins of the form $\mathcal{U}\left(\boldsymbol{C}_{0}^{\prime}, \Delta_{0}\right) * \mathcal{U}\left(\boldsymbol{C}_{1}^{\prime}, \Delta_{1}\right)$, where for $i=0,1, \ell_{i}$ is a building of type $\left(W_{T_{i}}, T_{i}\right)$ for $T_{i} \subset S_{i}$ and $\operatorname{dim} \Delta_{i}=\operatorname{Card}\left(T_{i}\right)-1$. If we choose $\ell_{0}$ to be the thin building $W_{0}$, then $W_{0} \square \ell_{1}$ can be regarded as the building with fundamental chamber the Coxeter cell $W_{0} K$ obtained, as in Example 2.6, by using the natural folding map from the Coxeter complex, $U\left(W_{0}, \Delta\right)$, to $\Delta$.

Example 3.10. (Some 3-dimensional hyperbolic reflection groups). Suppose that $\left(W_{0}, S_{0}\right)$ and $\left(W_{1}, S_{1}\right)$ are irreducible spherical Coxeter systems of rank 3 with diagrams either

$$
\mathbf{A}_{3} \circ \text { or } \circ \mathbf{B}_{3} \circ{ }^{4} \circ \text { or }
$$

Then $L_{0} \square L_{1}$ is an octahedron and $K:=K\left(L_{0} \square L_{1}\right)$ is isomorphic to a 3-dimensional cube. As in Example 3.2, to define $W_{0} \square W_{1}$, we need to choose a bijection $\theta_{01}: S_{0} \rightarrow S_{1}$. Suppose that $S_{0}=\left\{s_{0}, s_{1}, s_{2}\right\}, S_{1}=\left\{t_{0}, t_{1}, t_{2}\right\}$, where $s_{1}$ and $t_{1}$ 
correspond to the middle nodes of their diagrams. Choose $\theta_{01}$ so that $\theta_{01}\left(s_{1}\right) \neq t_{1}$. For such a choice of $\theta_{01}$ it follows from Andreev's Theorem [2] that $K$ can be realized as a convex polytope in hyperbolic 3 -space, $\mathbb{H}^{3}$, with dihedral angles between the faces as prescribed by the Coxeter matrix $(m(s, t))$ of $W_{0} \square W_{1}$. (In other words, the dihedral angle between the faces of corresponding to $s$ and $t$ is $\pi / m(s, t)$.) The group generated by the hyperbolic reflections across the faces of this polytope is isomorphic to $W_{0} \square W_{1}$. The condition that must be checked for Andreev's Theorem to hold is that there are no cycles (without "diagonals") of length 4of faces in $K$ with all dihedral angles $\pi / 2$, cf. [12], $\$ 6.10$. The polytope $K$ has two distinguished opposite vertices where the dihedral angles are determined by one of the above diagrams. Each such vertex is contained in one edge where the dihedral angle is $\pi / 2$. The condition that $\theta_{01}\left(s_{1}\right) \neq t_{1}$ means that these $\pi / 2$-edges are not opposite to each other. So, Andreev's Condition holds.

Next suppose that $W_{0}$ and $W_{1}$ are Euclidean reflection groups of rank 3. Then $K$ has the same set of faces as a "cube with two ideal vertices," i.e, two opposite vertices have been removed from the 3 -cube. As before we can choose $\theta_{01}$ to break up cycles of $\pi / 2$-edges. In a second paper, [3], Andreev generalized his theorem to cover polytopes with ideal vertices. This second result implies that we can realize the cube with ideal vertices as a convex polytope in $\mathbb{H}^{3}$ with two ideal vertices and with the prescribed dihedral angles. Hence, $W_{0} \square W_{1}$ is a reflection group on $\mathbb{H}^{3}$ with a fundamental polytope which is noncompact but which has finite volume.

Hyperbolic buildings. A building is hyperbolic if $W$ is a cocompact reflection group on $\mathbb{H}^{n}$. This implies that the building can be given a metric so that each apartment is isometric to $\mathbb{H}^{n}$, cf. [1], Chapter 12 .

A building is called Gromov hyperbolic if $W$ is word hyperbolic. Moussong [23] showed that if this is the case, then the standard realization $\mathcal{U}(W, K)$ can be given a CAT $(-1)$ metric, cf. [12], §12.6. It is proved in [11] that if the standard realization of an apartment is CAT $(-1)$, then so is the standard realization of a corresponding building. (For the definition of "CAT(-1)" see, for example, [12], Appendix I.)

It seems that examples of (thick) hyperbolic buildings previously have only been constructed in the following two cases:

- $W$ a 2-dimensional hyperbolic reflection group generated by the reflections across the edges of a convex polygon in $\mathbb{H}^{2}$ with angles integral submultiples of $\pi$. (These are called Fuchsian buildings.)

- $W$ is a right-angled Coxeter group, generated by reflections across the faces of a right-angled polytope in $\mathbb{H}^{n}$. (Such polytopes can only occur in dimensions $n \leq 4$.)

Examples of Fuchsian buildings can be constructed using the theory of Kac-Moody groups, e.g., in [18]. It is proved in [11] that for a right-angled $W$ one can construct buildings of any prescribed thickness along each mirror of $K$. For discussions of 
right-angled hyperbolic buildings in dimensions 2 and 3 see [5], [6], [7], [17], [18]. New examples of 3-dimensional hyperbolic buildings, which are not right-angled, are given below.

Example 3.11 (3-dimensional hyperbolic buildings). Let $\ell_{0}$ and $\mathcal{C}_{1}$ be finite spherical buildings of type $\mathbf{A}_{3}$ or $\mathbf{B}_{3}$. It follows from the discussion in Example 3.10 that $U\left(\ell_{0} \square \ell_{1}, K\right)$ is a hyperbolic building. The buildings $\ell_{0}$ and $\ell_{1}$ correspond to algebraic groups of rank 3 over finite fields, and the order of the fields can be different for $\ell_{0}$ and $\ell_{1}$. Similarly, by using the second paragraph of Example 3.10, one can construct buildings with fundamental polytope a combinatorial cube in $\mathbb{H}^{3}$ with two ideal vertices.

Example 3.12 (Free products of Gromov hyperbolic buildings). Suppose that we are given Gromov hyperbolic buildings, $\mathscr{C}_{0}, \ldots, \mathcal{C}_{p}$. It follows from [23] and [11] that the standard realization of the free product, $\ell_{0} * \cdots * \ell_{p}$, can be given a CAT( $(-1)$ metric and hence, is Gromov hyperbolic. Also note that spherical buildings are Gromov hyperbolic, so we could choose any of the $\mathcal{C}_{i}$ to be spherical.

\section{Questions related to Kac-Moody groups}

Suppose that $(W, S)$ is such that $m(s, t) \in\{2,3,4,6, \infty\}$ for all $s \neq t$ and that $q$ is a prime power. As in [29] one can choose an $(S \times S)$ "Cartan matrix" compatible with $(W, S)$ and from this construct a Kac-Moody Lie algebra and a Kac-Moody group $G$ over the field $\mathbb{F}_{q}$ of order $q$. Associated to $G$ there is a Tits system $(G, B, N, T)$ and a building $\mathcal{C}_{G}$ with set of chambers $G / B$. Call $\mathcal{C}_{G}$ a Kac-Moody building.

Now suppose as in $\S 2$ or $\S 3$ that $\ell^{\prime}$ is a spherical building of type $\left(W^{\prime}, S^{\prime}\right)$ associated to an algebraic group $G^{\prime}$ over a finite field $\mathbb{F}_{q}$. Then $(W, S)$ satisfies the crystallographic condition (that $m(s, t) \in\{2,3,4,6, \infty\}$ ). Let $\mathcal{C}$ be the building constructed in $\S 2$. The question arises: is $\mathcal{C}$ isomorphic to a Kac-Moody building $\ell_{G}$ ? Undoubtedly, it often is. For example, if $(W, S)$ is a right-angled and $\ell_{\text {is }}$ is thickness $q$ (so that $G^{\prime}$ is the $S^{\prime}$-fold product, $\prod \operatorname{PGL}\left(2, \mathbb{F}_{q}\right)$ ), then $\mathscr{C} \cong \mathscr{C}_{G}$ (since any two regular right-angled buildings of type $(W, S)$ and the same thickness $q$ are isomorphic). However, even when $\mathscr{C} \cong \mathscr{C}_{G}$, the group $\widetilde{G} \subset \operatorname{Aut}(\mathcal{C})$, obtained by lifting the $G^{\prime}$-action to $\mathcal{C}$, will not be closely related to the Kac-Moody group $G$. One reason is that if $(W, S)$ is irreducible, infinite and nonaffine, then, by a result of Caprace-Remy [9], $G$ is a simple group. On the other hand, there is an epimorphism $\widetilde{G} \rightarrow G^{\prime}$. Hence, the restriction of this epimorphism to $\widetilde{G} \cap G$ is either trivial or else it does not extend to $G$. (Further information about right-angled buildings and Kac-Moody buildings can be found in Rémy-Ronan [24].) 


\section{References}

[1] P. Abramenko and K. S. Brown, Buildings. Grad. Texts in Math. 248, Springer-Verlag, New York 2008. Zbl 05288866 MR 2439729

[2] E. M. Andreev, On convex polyhedra in Lobačevskiǔ spaces. Mat. Sb. (N.S.) 81 (123) (1970), 445-478; English transl. Math. USSR-Sb. (N.S.) 10 (1970), 413-440. Zbl 0194.23202 MR 0259734

[3] E. M. Andreev, On convex polyhedra of finite volume in Lobačevskiǐ space. Mat. Sb. (N.S.) 83 (125) (1970), 256-260. English transl. Math. USSR-Sb. (N.S.) 12 (1970), 255-259. Zbl 0203.54904 MR 0273510

[4] A. Barnhill and A. Thomas, Density of commensurators for uniform lattices of rightangled buildings. Preprint 2008. arXiv:0812.2280

[5] M. Bourdon, Immeubles hyperboliques, dimension conforme et rigidité de Mostow. Geom. Funct. Anal. 7 (1997), 245-268. Zbl 0876.53020 MR 1445387

[6] M. Bourdon, Sur les immeubles fuchsiens et leur type de quasi-isométrie. Ergodic Theory Dynam. Systems 20 (2000), 343-364. Zbl 0965.53030 MR 1756974

[7] M. Bourdon and H. Pajot, Rigidity of quasi-isometries for some hyperbolic buildings. Comment. Math. Helv. 75 (2000), 701-736. Zbl 0976.30011 MR 1789183

[8] N. Bourbaki, Lie groups and Lie algebras. Chapters 4-6. Springer-Verlag, Berlin 2002. Zbl 0983.17001 MR 1890629

[9] P.-E. Caprace and B. Rémy, Simplicité abstraite des groupes de Kac-Moody non affines. C. R. Acad. Sci. Paris Sér. I Math. 342 (2006), 539-544. Zbl 1095.22003 MR 2217912

[10] M. W. Davis, Some aspherical manifolds. Duke Math. J. 55 (1987), 105-139. Zbl 0631.57019 MR 883666

[11] M. W. Davis, Buildings are CAT(0). In Geometry and cohomology in group theory (Durham, 1994), London Math. Soc. Lecture Note Ser. 252, Cambridge University Press, Cambridge 1998, 108-123. Zbl 0978.51005 MR 1709955

[12] M. W. Davis, The geometry and topology of Coxeter groups. London Math. Soc. Monogr. 32, Princeton University Press, Princeton, NJ, 2007. Zbl 1142.20020 MR 2360474

[13] M. W. Davis, J. Dymara, T. Januszkiewicz, J. Meier, and B. Okun, Compactly supported cohomology of buildings. To appear in Comment. Math. Helv., arXiv:0806.2412

[14] M. W. Davis and T. Januszkiewicz, Right-angled Artin groups are commensurable with right-angled Coxeter groups. J. Pure Appl. Algebra 153 (2000), 229-235. Zbl 0982.20022 MR 1783167

[15] M. W. Davis and J. Meier, The topology at infinity of Coxeter groups and buildings. Comment. Math. Helv. 77 (2002), 746-766. Zbl 1022.20016 MR 1949112

[16] M. W. Davis and J. Meier, Erratum to "The topology at infinity of Coxeter groups and buildings". Comment. Math. Helv. 82 (2007), 235-236. MR 2296063

[17] J. Dymara and D. Osajda, Boundaries of right-angled hyperbolic buildings. Fund. Math. 197 (2007), 123-165. Zbl 05221117 MR 2365885 
[18] D. Gaboriau and F. Paulin, Sur les immeubles hyperboliques. Geom. Dedicata 88 (2001), 153-197. Zbl 1020.51007 MR 1877215

[19] F. Haglund, Existence, unicité et homogénéité de certains immeubles hyperboliques. Math. Z. 242 (2002), 97-148. Zbl 1052.51009 MR 1985452

[20] F. Haglund, Commensurability and separability of quasiconvex subgroups. Algebr. Geom. Topol. 6 (2006), 949-1024. Zbl 05118551 MR 2240922

[21] F. Haglund and F. Paulin, Constructions arborescentes d'immeubles. Math. Ann. 325 (2003), 137-164. Zbl 1025.51014 MR 1957268

[22] T. Januszkiewicz and J. Świątkowski, Commensurability of graph products. Algebr. Geom. Topol. 1 (2001), 587-603. Zbl 0998.20029 MR 1875609

[23] G. Moussong, Hyperbolic Coxeter groups. Ph.D. thesis, The Ohio State University, Columbus 1988. http://www.ohiolink.edu/etd/view.cgi?osu1114437114

[24] B. Rémy and M. Ronan, Topological groups of Kac-Moody type, right-angled twinnings and their lattices. Comment. Math. Helv. 81 (2006), 191-219. Zbl 05033719 MR 2208804

[25] M. Ronan, Lectures on buildings. Perspect. Math. 7, Academic Press Inc., Boston 1989. Zbl 0694.51001 MR 1005533

[26] A. Thomas, Lattices acting on right-angled buildings. Algebr. Geom. Topol. 6 (2006), 1215-1238. Zbl 1128.22002 MR 2253444

[27] A. Thomas, On the set of covolumes of lattices for Fuchsian buildings. C. R. Acad. Sci. Paris Sér. I Math. 344 (2007), 215-218. Zbl 1150.20013 MR 2292989

[28] J. Tits, Buildings and group amalgamations. In Proceedings of groups - St Andrews 1985, London Math. Soc. Lecture Note Ser. 121, Cambridge University Press, Cambridge 1986, 110-127. Zbl 0631.20019 MR 0896503

[29] J. Tits, Uniqueness and presentation of Kac-Moody groups over fields. J. Algebra 105 (1987), 542-573. Zbl 0626.22013 MR 873684

[30] A. Vdovina, Combinatorial structure of some hyperbolic buildings. Math. Z. 241 (2002), 471-478. Zbl 1020.51009 MR 1938699

[31] X. Xie, Quasi-isometric rigidity of Fuchsian buildings. Topology 45 (2006), 101-169. Zbl 1083.51008 MR 2170496

Received December 1, 2008; revised February 7, 2009

M. W. Davis, Department of Mathematics, Ohio State University, 231 W. 18th Ave.,

Columbus, OH 43210, U.S.A.

E-mail: mdavis@math.ohio-state.edu 